\title{
El Mecanismo Europeo de Estabilidad: uno de los pilares del modelo de gobernanza económica de la UE y su déficit de legitimidad
}

Marcin Roman Czubala*

\section{RESUMEN}

El establecimiento del MEDE, un organismo que facilita la ayuda financiera bajo unas condiciones estrictas, proporcionando de este modo el apoyo a la estabilidad de la zona euro, fue una clara respuesta de la Unión Europea ante la crisis económica vigente.

Dicha entidad, que forma parte del actual modelo de gobernanza económica de la UE, es un elemento clave en el proceso de coordinación y vigilancia económica reforzadas, lo que hace que su estudio se considere de máxima relevancia y actualidad.

Por eso, el principal objetivo de este artículo consiste en examinar su estructura jurídica, la configuración, el modelo de financiación y la capacidad de actuación, para poder analizar posteriormente su viabilidad y el grado de déficit de legitimidad que presenta.

Palabras clave: crisis económica, déficit de legitimidad, gobernanza económica, Mecanismo Europeo de Estabilidad, Unión Europea.

* Máster en Política Internacional: Estudios Sectoriales y de Área. Investigador de la Facultad de Ciencias Políticas y Sociología, Universidad Complutense, Madrid (España).mczubala@ucm.es

Recibido: 29 de marzo de 2015 / Aceptado: 6 de junio de 2015.

Para citar este artículo

Czubala, M. R. (2015). El Mecanismo Europeo de Estabilidad: uno de los pilares del modelo de gobernanza económica de la UE y su déficit de legitimidad. OASIS, 21, 133-145. DOI: http://dx.doi.org/10.18601/16577558.n21.07 
The European Stability Mechanism: one of the pillars of the EU's model of economic governance and its legitimacy deficit

\section{ABSTRACT}

The establishment of the ESM, the entity that offers financial assistance under strict conditions with the purpose of providing support for the stability of the euro area, was a clear European Union response to the current economic crisis.

This entity, which is a part of the current model of economic governance of the $\mathrm{EU}$, is a key element in the process of its coordination and improved economic surveillance, which makes its study to be considered highly relevant and current.

Therefore, the main goal of this article is to examine its legal structure, configuration, funding model, and ability to act, in order to then assess its feasibility and the degree of legitimacy deficit that it presents.

Key words: Economic crisis, economic governance, European Stability Mechanism, European Union, legitimacy deficit.

\section{INTRODUCCIÓN}

A raíz de la progresiva profundización en el proceso de integración europea, los vínculos entre los Estados miembros, tanto en el sentido político como económico y social, se han estrechado. Este proceso, llevado a cabo durante todos estos años, ha sido el motor determi- nante en clave europea. Asimismo, permitió adoptar un conjunto de medidas y reformas con el objetivo de responder a los múltiples retos marcados a los países miembros en su conjunto a lo largo de este tiempo. Por otro lado, no cabe duda de que es ahora cuando la Unión Europea atraviesa uno de los momentos más importantes en su historia, no solamente difíciles, sino también decisivos para su futura evolución.

La actual crisis económica, presupuestaria y financiera ha puesto en evidencia los fallos del sistema establecido, así como la enorme diversidad de los modelos económicos nacionales en el marco de la UE. Asimismo, ha subrayado la necesidad de establecer un nuevo modelo de gobernanza económica dotado de instituciones y procedimientos adecuados con el fin de paliar los efectos negativos de la crisis y devolver la estabilidad macroeconómica al continente, además de progresar en el contexto de la integración política.

Infortunadamente, el conjunto de medidas aplicadas, basadas en mayor coordinación y vigilancia de las políticas presupuestarias y macroeconómicas al nivel europeo, no causó el efecto esperado, tanto en el crecimiento de los países miembros de la zona euro con problemas de la deuda soberana, como en la mejora notable del estado económico del conjunto de la Unión Europea. Por eso, la hipótesis principal de este trabajo está basada en la premisa de que la creación de un sistema de gobernanza económica sólido y persistente, con el fin de nivelar las desventajas provocadas por la crisis, es de máxima actualidad y relevancia.

El objetivo de este artículo es situar el debate acerca de este tema a partir del análisis del 
Mecanismo Europeo de Estabilidad (MEDE), es decir, uno de los tres pilares de la gobernanza económica comunitaria actual y de gestión de la crisis de la eurozona, sin entrar en el estudio de los instrumentos de carácter temporal como la Facilidad Europea de Estabilidad Financiera y el Mecanismo Europeo de Estabilidad que este sustituyó.

Para llevarlo a cabo, primero se presentará un marco teórico. En segundo lugar, se examinará la estructura jurídica, la configuración, el modelo de financiación y la capacidad de actuación del dicho mecanismo. Posteriormente, se evaluará su viabilidad y legitimidad institucional, su impacto sobre el diálogo entre la Unión Europea y la sociedad, así como su existencia en el marco de la prevención y resolución de la crisis de la UE. Por último, se expondrán los resultados obtenidos.

\section{MARCO TEÓRICO}

La tradición liberal, basada en el énfasis sobre el Estado-nación, sufrió un cambio importante a raíz de los distintos procesos de regionalización y globalización del siglo xx. La introducción de los diferentes cambios en los sistemas de gobernanza, fue uno de los factores clave de lo que conocemos hoy como la crisis de la democracia.

Igualmente, el cuestionamiento de la responsabilidad y el control político vinculados a este proceso llevan al desarrollo de una serie de estudios que analizan los conceptos de privatización, ingobernabilidad, crisis de Estado de derecho y poder invisible desarrollados, entre otros, por Held (1995). El replanteamiento de la democracia y de los agentes transnacionales como fuente de la gobernanza global permite establecer diferentes percepciones sobre este tema.

Por un lado, autores como Dahl (1999) subrayan la dificultad de la participación ciudadana, la rendición de cuentas, la atención a los Estados de bienestar y protección de las libertades individuales ante la desaparición de las fronteras nacionales, cuestionando el consentimiento ciudadano como una forma de legitimar las acciones de los gobernantes. Por otro lado, podemos destacar a Habermas (1975), que entiende la crisis de legitimidad como un efecto de los acontecimientos económicos que causan una brecha creciente entre la sociedad y los diferentes aspectos procedimentales de la democracia.

De esta forma, podemos decir que la Unión Europea es un claro ejemplo de la gobernanza global. Además, esta organización internacional cambiante tiene la necesidad de responder a los retos de un modelo de democracia en profundo cambio, siendo un proyecto vinculado a los ciudadanos de sus Estados miembros. Tal como lo establece Weber (1921), la legitimidad de la UE es de carácter legal. Es decir, está basada en la aprobación de los Tratados por parte de los gobiernos, a través de los cuales se establecen los compromisos políticos entre sus diferentes miembros.

Dicha concepción, estudiada también por diferentes autores como Lipset (1963), Habermas (1975), Rogowski (1974), O'Kane (1993) o Beetham (1991), nos lleva al trabajo de Scharpf (2000), en el que dicho autor centra el problema de la legitimidad democrática de la UE en un cuestionamiento a la legitimidad de origen (input), entendida por el conjunto de las 
elecciones políticas a partir de las preferencias de los miembros de la comunidad y la creencia en una identidad colectiva. Tal como lo explica Castells (1999), la crisis de la democracia es la causante, no solamente de la deslegitimación, sino también de la crisis de la credibilidad.

Es importante subrayar el trabajo de Moravcski (2002), en el que el autor centra su estudio sobre la relevancia de la legitimidad de resultado (out), sustentándola en la resolución de los problemas planteados con el objetivo de generar bienestar común de los ciudadanos. Moravcski (2004), predica también que el aislamiento y la autonomía de los órganos políticos son legítimos y que incluso este proceso de delegación de algunas políticas a los órganos sin responsabilidad democrática puede aumentar la legitimidad, fundamentalmente derivada de los siguientes aspectos. Primero, la nivelación de la vulnerabilidad de las políticas a las presiones nacionales evitando la "tiranía de la mayoría” y los intereses especiales. Segundo, la mejora de las competencias de los gestores políticos convertidos en "los gobiernos de expertos", así como la ignorancia racional de los votantes, llevando de esta forma al ahorro de costes en la adopción de decisiones.

Sin embargo, es esta delegación de funciones de los Estados miembros a instituciones supranacionales lo que lleva a autores como Scharpf (2005), Follesdal y Hix (2006) a argumentar que la gobernanza de la UE tiene un déficit democrático donde la responsabilidad, el control político y la participación ciudadana son estándares de esta crítica.

Otro de los aspectos por analizar es la legitimidad formal, es decir, la cuestión de la responsabilidad y rendición de cuentas. Majone (2003) establece que las instituciones sobre las que se delega se aíslan del control político y democrático para darles mayor eficiencia y credibilidad a los compromisos políticos a largo plazo. De esta forma, los ciclos de alternancia democrática están limitados temporalmente y condicionan la toma de decisiones a corto plazo.

La teoría de la delegación en instituciones no mayoritarias, desarrollada en los trabajos de Majone (1996), Pollack $(1997,2003)$ y Tallberg $(2000,2002)$, establece también que los gobernantes deciden delegar funciones en agentes solamente si los beneficios esperados superan los costes. Basándonos en el estudio de Thatcher y Stone Sweet (2002), podemos distinguir tres tipos de beneficios: la credibilidad de las promesas realizadas a los ciudadanos, la necesidad de políticas de expertos que resuelvan las asimetrías de información en áreas técnicas de la gobernanza, así como la maximización de los objetivos políticos de los agentes gracias a las políticas impopulares para ciertos sectores de la sociedad.

En el caso de la UE, la aplicación de este análisis es aún más compleja porque a la delegación a nivel comunitario, dentro de las instituciones comunitarias, se añade el problema de la delegación a nivel externo (transferencia de poder de decisión a instituciones independientes).

Con el fin de paliar el déficit democrático presente en el contexto de la Unión Europea, se han introducido diferentes cambios en el marco del Tratado de Lisboa. Entre los más destacables se encuentran el fomento a la participación más directa de los parlamentos nacionales, así como la ampliación del papel ejercido por el Parlamento Europeo. Sin em- 
bargo, el método de la aprobación del Tratado es, en sí mismo, un ejemplo claro de déficit democrático, siendo este un parche temporal a la imposibilidad de dotar a la UE de una constitución para los ciudadanos de la Unión.

\section{MECANISMO EUROPEO DE ESTABILIDAD - EL ESTADO DE LA CUESTIÓN}

La firma del acuerdo suscrito por los dirigentes de Francia y Alemania, Nicolas Sarkozy y Angela Merkel, en Deauville el 18 de octubre de 2010, abrió un largo debate acerca de la necesidad del establecimiento y la configuración del futuro MEDE. El hecho de que la efectividad de las acciones aprobadas hasta entonces en materia de nivelación de los efectos negativos de la crisis hubiera sido muy baja, hizo que la primera economía europea se mostrase favorable a la creación de un mecanismo permanente que pudiera actuar de modo eficiente contra esta situación.

La posterior convicción del resto de los países pertenecientes a la zona euro se concretó con base en una propuesta aprobada en la reunión del Consejo Europeo del 28 y 29 de octubre de 2010. Los Estados miembros expresaron su voluntad acerca de la creación de tal mecanismo con el objetivo de asegurar la estabilidad financiera de la eurozona en su conjunto, así como de describir las bases de su funcionamiento.

Más adelante, en la reunión del 16 y 17 de diciembre de 2010, el Consejo Europeo no solamente pidió al Eurogrupo y a la Comisión Europea que preparasen un acuerdo intergubernamental antes de marzo de 2011, sino que acordó también que el futuro mecanismo iba a sustituir, a partir del 1 de julio de 2013, a los instrumentos de carácter temporal como la Facilidad Europea de Estabilidad Financiera y el Mecanismo Europeo de Estabilidad Financiera, convirtiéndose en un ente único a la hora de facilitar los futuros rescates, lo que implicaría, además, cambios en el texto del Tratado de Lisboa.

Tras la reunión de los ministros de finanzas del 22 de marzo de 2011, el Consejo Europeo aprobó la Decisión 2011/199/UE afirmando la mayoría de las reglas de funcionamiento del MEDE acordadas anteriormente, además de llegar a un consenso político acerca de la modificación del artículo 136 del Tratado de Funcionamiento de la Unión Europea, ampliándolo con un apartado nuevo.

Asimismo, el Tratado Constitutivo del MEDE fue firmado el 11 de julio de 2011. Aun así, y debido a la necesidad de introducir ciertas modificaciones en el texto aprobado, incluidas las decisiones tomadas en la segunda mitad del 2011 sobre la mejora de la eficacia del MEDE y la aceleración de la realización de sus acciones, el día 2 de febrero de 2012 se procedió a la firma de su segunda y definitiva versión. Por último, su puesta en marcha se dio el día 8 de octubre de ese mismo año.

Al día de hoy, el Mecanismo es un organismo de carácter intergubernamental de derecho internacional público, con sede en Luxemburgo. Su función principal consiste en facilitar la ayuda financiera, bajo unas condiciones estrictas, proporcionando de este modo el apoyo a la estabilidad de la zona euro en su conjunto y de sus países miembros. Además, la adopción del euro por un Estado 
miembro lo convierte de modo automático en nuevo miembro del MEDE, obligándolo a asumir todos los derechos y las obligaciones correspondientes.

El Consejo de Gobernadores, el principal órgano de control y supervisión del MEDE, está compuesto por los ministros de Economía y Finanzas de los Estados miembros de la eurozona, quienes poseen derecho al voto. En caso de ausencia de alguno de ellos, el respectivo puesto se cubre con el gobernador suplente designado con anterioridad por su país miembro. Igualmente, los mencionados nombramientos son revocables en todo momento. Además, el presidente y el vicepresidente del Consejo de Gobernadores se eligen entre y por los miembros con derecho a voto por un periodo de dos años con una posibilidad de reelección.

En las reuniones del órgano analizado participan también, aunque solamente en condición de observadores, el comisario europeo de Asuntos Económicos y Monetarios, el presidente del Banco Central Europeo, así como el presidente del Eurogrupo en el caso de que no ejerza de presidente o de gobernador. En cuanto a los representantes de los Estados miembros no pertenecientes a la zona euro, los que participen de forma ad hoc en el proceso de estabilización a favor de un país de la eurozona podrán estar invitados como observadores a las reuniones respectivas de este órgano. Por último, los representantes de las otras entidades, como las organizaciones internacionales, pueden ser invitados de forma puntual y sin derecho a voto.

La proporción de la asistencia financiera, el establecimiento de las condiciones de la mencionada asistencia, la capacidad crediticia del MEDE, así como los cambios en la gama de actividades de los instrumentos, son las funciones principales del Consejo de Gobernadores (Banco Central Europeo, 2011, p. 80). Las decisiones en el contexto de estas cuatro materias tienen que ser adoptadas por unanimidad, teniendo en cuenta que las posibles abstenciones no impiden de ningún modo que se adopte la decisión. Mientras tanto, el resto de las decisiones requieren una mayoría cualificada (el $80 \%$ de los votos ponderados proporcionales a la contribución de cada Estado miembro de la eurozona al ME$\mathrm{DE})$, salvo que se establezca otra cosa. Por otro lado, se informará también al PE sobre todas las operaciones que se lleven a cabo en su marco, asegurando de este modo una auditoría tanto de carácter interno, como externo.

Otro de los órganos principales del MEDE es el Consejo de Administración, compuesto por los administradores y sus suplentes, ambos nombrados por cada uno de los Estados miembros del Eurogrupo, que serán revocables en todo momento. La toma de decisiones en el marco de esta institución requiere una mayoría cualificada (la misma que del anterior órgano), salvo que se disponga otra cosa, llevando a cabo de este modo los cometidos delegados por el Consejo de Gobernadores.

Igualmente, el comisario europeo de Asuntos Económicos y Monetarios, y el presidente del Banco Central Europeo tienen la posibilidad de nombrar a un observador cada uno. Por otro lado, los Estados miembros que no forman parte de la eurozona, así como los representantes de otros entes, podrán participar en condición de observadores de acuerdo con los mismos requisitos que en el caso del Consejo de Gobernadores. 
Este mismo órgano (el Consejo) nombra también al director ejecutivo del MEDE, cuyo mandato será de cinco años con posibilidad de ser renovado una sola vez. Durante su jefatura, el director tiene la obligación de presidir el Consejo de Administración, además de participar en las reuniones del Consejo de Gobernadores y llevar a cabo la gestión corriente del MEDE.

En cuanto a la actuación del MEDE, esta puede tomar una de las siguientes formas (MEDE, 2012):

- Préstamos a los Estados que se encuentren en una situación de graves problemas de financiación o corran alto riesgo de los mismos.

- Compra de los bonos de un país miembro de la eurozona en los mercados primarios y secundarios de deuda.

- Línea de crédito.

- Recapitalización financiera de las instituciones financieras con base en préstamos concebidos a través de los Estados.

La ayuda otorgada está vinculada a un riguroso plan de ajuste macroeconómico y su posterior implementación. El tipo de interés aplicado, sea fijo o variable, es revisado regularmente por el Consejo de Gobernadores. Asimismo, al capital concebido se le ańadirán los cien puntos básicos en el caso de no pago durante los primeros tres ańos, aparte de los doscientos puntos básicos que conllevará la aplicación de cada una de estas operaciones desde su principio.

Es importante destacar que su petición no debe resultar atractiva al país miembro en cuestión. Esta situación se debe principalmente a la compensación del riesgo asumido por los Estados que forman parte del mecanismo analizado, así como a la reducción del posible riesgo moral. En cuanto a las condiciones de compra de los bonos de un país miembro, estas estarán vinculadas a la peculiaridad de la situación en la que se encuentre, así como a la misma condicionalidad macroeconómica de los préstamos.

Además, debido a la complejidad de los cambios que surgen a diario en el escenario internacional, se permitió que el Consejo de Gobernadores, bajo mutuo acuerdo, pueda ampliar el abanico de los instrumentos del Mecanismo teniendo como principal objetivo el aumento de su eficacia y la reducción de los efectos negativos de la crisis.

Para la activación del MEDE es necesaria una petición proveniente de un país perteneciente a la zona euro transferida al director gerente del Fondo Monetario Internacional (FMI), el presidente del Consejo Económico y Financiero y el del Eurogrupo. Tras dar este paso inicial, son la Comisión Europea, junto con el Fondo Monetario Internacional y el Banco Central Europeo, quienes estudian detalladamente la situación en la que se encuentra dicho Estado miembro y si existe algún tipo de riesgo para la estabilidad financiera para la Eurozona. Hay que subrayar que tanto el BCE como el FMI desempeñan un papel al nivel técnico en el proceso de la concesión de la ayuda financiera, pero solamente el último de ellos actúa también al nivel financiero dentro del proceso descrito.

En el caso de que la sostenibilidad de la deuda pública del país en cuestión cree una situación de riesgo, se analizan las necesidades de financiación del Estado solicitante, procediendo posteriormente a las negociaciones de 
un programa de ajuste que forma parte del Convenio de Cooperación final. La función de establecer las condiciones y conceder los fondos está en manos del Consejo de Gobernadores, mientras que es el Consejo de la Unión Europea el que refrenda la anterior propuesta de la Comisión.

La recepción del préstamo desde el MEDE implica también el involucramiento del sector privado del país receptor, entre otros, con el objetivo de fomentar la presión sobre este para cumplir los requisitos marcados en el plan de ajuste macroeconómico. Asimismo, el alcance de la participación de dichas entidades estará condicionado por la situación de cada Estado miembro, el nivel de sostenibilidad de su deuda o las implicaciones para la estabilidad de la eurozona (Martínez y Calvo, 2012, p. 34).

El proceso de supervisión, llevado a cabo por parte de la troika, consistirá en examinar la correcta aplicación del programa de ajunte mencionado, así como informar al Consejo de Administración y al Consejo ECofin de los resultados obtenidos. Es el Consejo quien, con base en estos informes, decide desembolsar o no los siguientes tramos de ayuda, disponiendo de la posibilidad de posponerlos o incluso suspenderlos en el caso de que no se cumplan las condiciones del programa. Igualmente, el seguimiento descrito puede prolongarse incluso a la fase posterior de la aplicación del programa, haciendo que este se mantenga hasta el momento de reintegración de la cantidad de ayuda financiera suficiente (Banco Central Europeo, 2011, p. 82).

El capital total previsto del MEDE, ampliado tras la entrada de Letonia a la zona euro, es de unos 705 mil millones de euros, de los cuales 80 mil millones están recaudados merced a desembolsos por parte de los Estados miembros del Eurogrupo de forma progresiva, mientras que los otros 625 mil millones de euros tienen carácter de capital exigible y de garantías de los miembros de la Eurozona. De este modo, la capacidad de préstamo efectiva del MEDE es de unos 500 mil millones de euros.

Los Estados miembros del MEDE establecieron también el margen de actuación para que el ratio entre el capital desembolsado y el saldo vivo del Mecanismo no sea menor al $15 \%$. El principal objetivo de esta decisión es garantizar su solidez y generar los estímulos económicos necesarios. Además, tal como se ha establecido desde el principio, dicha actuación se aplicará en el caso de necesidad y siempre a partir de julio de 2013.

Por otro lado, la contribución de los Estados miembros al MEDE está basada en el sistema de sus participaciones en el capital del Banco Central Europeo. En el caso de que el PIB per cápita de un Estado miembro perteneciente al Eurogrupo sea inferior al $75 \%$ de la media de la Unión Europea, se aplicaría una corrección de carácter temporal por un periodo máximo de doce meses después de que aquel adopte la moneda única o la entrada en vigor del MEDE. 
TABLA 1

LA CONTRIBUCIÓN DE LOS ESTADOS MIEMBROS DE LA EUROZONA AL MEDE

\begin{tabular}{|l|c|}
\hline \multicolumn{1}{|c|}{$\begin{array}{c}\text { Estados miembros } \\
\text { de la zona euro }\end{array}$} & $\begin{array}{c}\text { Participación en el } \\
\text { presupuesto del MEDE (\%) }\end{array}$ \\
\hline $\begin{array}{l}\text { República Federal } \\
\text { de Alemania }\end{array}$ & 26,9616 \\
\hline República Francesa & 20,2471 \\
\hline República Italiana & 17,7917 \\
\hline Reino de España & 11,8227 \\
\hline Reino de los Países Bajos & 5,6781 \\
\hline Reino Unido de Bélgica & 3,4534 \\
\hline República Helénica & 2,7975 \\
\hline República de Austria & 2,7644 \\
\hline República Portuguesa & 2,4921 \\
\hline República de Finlandia & 1,7852 \\
\hline República de Irlanda & 1,5814 \\
\hline República Eslovaca & 0,8184 \\
\hline República de Eslovenia & 0,4247 \\
\hline República de Lituania & 0,4063 \\
\hline República de Letonia & 0,2746 \\
\hline Gran Ducado de & 0,2487 \\
\hline Luxemburgo & $\mathbf{1 0 0 , 0}$ \\
\hline República de Chipre & 0,1847 \\
\hline República de Estonia & 0726 \\
\hline República de Malta & \\
\hline Total & \\
\hline
\end{tabular}

Fuente: elaboración propia a partir de http://www.esm.europa.eu/about/ governance/shareholders/index.htm

Finalmente, no hay que olvidar que la Facilidad Europea de Estabilidad Financiera mantendrá su actuación debido a la existencia y la necesidad de finalización de los programas que comenzaron antes de la firma del Tratado Constitutivo del Mecanismo. Sin embargo, los Estados miembros acordaron que hasta julio de 2013, la FEEF también formaría parte de los distintos programas nuevos, asegurando de este modo la plena capacidad prestataria del MEDE. Asimismo, pasada esta fecha, se prolongará su actuación hasta que todos los préstamos y bonos concebidos hayan sido pagados.

\section{RESULTADOS Y DISCUSIÓN - LA VIABILIDAD Y LA LEGITIMIDAD DEL MEDE}

Al hablar de la viabilidad y legitimidad institucional del MEDE, es necesario subrayar una serie de características, siendo estas el resultado del carácter intergubernamental y el estatus legal que presenta.

Primero, la toma de decisión y la supervisión del MEDE residen en manos de los Estados miembros con derecho a voto. En otros términos, todos los países pertenecientes a la zona euro que al mismo tiempo forman parte del Consejo de Gobernadores. Esta situación, claro resultado de los efectos negativos de la crisis y de la enorme necesidad de actuación, abre la posibilidad de estrechar la cooperación en el marco de un grupo más reducido que la Unión Europea en su conjunto.

Asimismo, el sistema de ponderación de votos y de aplicación de las medidas macroeconómicas sobre los beneficiarios de la ayuda crean una situación de desigualdad, ya que algunos de los Estados miembros guardan claramente más poder de decisión que otros, siendo capaces de decidir acerca del destino económico de los demás. En el caso de una votación en la que se requiera una mayoría cualificada, se puede dar la situación en la que una coalición de al menos cuatros países pueda decidir por el resto de los Estados. Además, 
solamente dos de ellos (Alemania y Francia) disponen de derecho de veto.

En el caso de un conflicto sobre la interpretación o aplicación del Tratado constitutivo del Mecanismo, será el Consejo de Gobernadores el que tendrá la potestad de resolverlo. En una situación en la que un país miembro o grupo de países afectados se opusieran a la resolución dictada, la controversia sería sometida al Tribunal de Justicia de la Unión Europea.

Segundo, las propiedades y los activos del MEDE no pueden ser intervenidos, confiscados o embargados, ni tampoco ser objeto de algún tipo de acción legal en su contra, salvo que se decida otra cosa. Igualmente, sus archivos y documentos oficiales también gozarán de inviolabilidad, impidiendo cualquier tipo de investigación profesional por parte de los tribunales, mientras que los altos cargos y demás empleados serán exentos de un proceso legal por las actividades ejercidas en su marco. El conjunto de estas características provoca una situación de completa inmunidad del MEDE frente a los poderes estatales de los países miembros, debilitando las democracias nacionales.

Desde otro punto de vista, no hay que olvidar que este tipo de soluciones son muy frecuentes en las entidades financieras de carácter internacional de este calibre (p. ej. el Fondo Monetario Internacional o el Banco Mundial). Además, el rango de inmunidad de sus altos cargos es de alguna forma proporcional al de los diplomáticos nacionales, lo que se debe en gran medida a las actividades y responsabilidades que desempeñan.

Tercero, el establecimiento y las operaciones realizadas en el marco del MEDE conllevarán una información periódica al Parlamento Europeo y los respectivos Parlamentos nacionales. Aun así, estos no podrán influir de forma directa sobre las decisiones tomadas en su marco.

Además, toda la actividad del organismo intergubernamental analizado será inspeccionada por una auditoría tanto interna como externa. No obstante, esta última deja mucho qué desear, ya que las entidades de auditoría independientes, responsables de realizarla (que tendrán además el acceso pleno a la documentación requerida), deben ser aprobadas antes por el Consejo de Gobernadores.

Por otro lado, se publicará un informe anual de la actividad del MEDE, en el que se integrará el estado de cuentas revisado, exponiéndolo no solamente a la disposición de los Parlamentos nacionales y los órganos supremos de auditoría de los países miembros del eurogrupo, sino también del Tribunal de Cuentas Europeo. Por último, se dará a conocer a los Estados un resumen trimestral acerca de la posición financiera que contendrá además la balanza de pagos del Mecanismo.

En cuanto al impacto del diálogo entre la Unión Europea y la sociedad, hay que destacar la enorme falta de un verdadero debate en el espacio público europeo acerca de la existencia y la configuración del MedE. Asimismo, la carencia de publicaciones académicas, la incomprensibilidad de la información disponible, así como la inexistencia de una vía fácil para su obtención, tampoco es indiferente a la cuestión. Todos estos factores, junto con la poca relevancia mediática que ha recibido el MEDE, hacen que la posibilidad de influir sobre su estructura de organización y su actuación por parte de la sociedad, de forma directa o a 
través de las instituciones democráticas, haya sido reducida significativamente.

Por último, y debido a los cambios que surgen en el escenario internacional, la existencia del MEDE en el marco de la prevención y resolución de la crisis de la UE tiene que ser reforzada. El Consejo de Gobernadores debe, bajo el mutuo acuerdo de todos los Estados miembros que lo componen, ampliar la gama de instrumentos y el presupuesto del MEDE con el objetivo de aumentar su eficacia y combatir los efectos negativos de la crisis.

De la misma manera, es necesario aplicar en su totalidad las medidas de prevención y de supervisión, permitiendo de este modo que los países miembros de la zona euro no tengan que recurrir a la intervención del Mecanismo.

Aún así, no hay que olvidar que el poder de los parlamentos de algunos de los Estados miembros (p. ej. Cámara baja de la Asamblea alemana, Bundestag) puede causar resultados contrarios al objetivo marcado, sobre todo en las situaciones caracterizadas por los grandes descontentos sociales con el proyecto europeo, así como por la mayor representación de los euroescépticos o altereuropeístas en las cámaras nacionales.

\section{CONCLUSIONES}

La actual crisis económica, financiera y presupuestaria ha demostrado una clara ineficacia del sistema de gobernanza económica comunitario existente. La gran diversidad de los modelos económicos de los Estados miembros de la Unión Europea y de los cambios surgidos en el contexto internacional tampoco han sido indiferentes. Igualmente, las reformas introducidas a partir del año 2011, no lograron los objetivos esperados, es decir, paliar los efectos negativos de la crisis, así como devolver la plena estabilidad macroeconómica al continente.

El Mecanismo Europeo de Estabilidad, un claro ejemplo de la respuesta a la situación económica actual y un paso más hacia el pleno cumplimiento de la unión económica y monetaria de la UE, es un organismo de carácter intergubernamental que otorga la ayuda a los Estados miembros de la eurozona con el fin de asegurar la estabilidad en su conjunto. La asistencia financiera concedida, a partir de los instrumentos de los que dispone, está vinculada a la aplicación y el posterior cumplimiento de un estricto programa de ajuste macroeconómico, siendo el medio de implementación de las reformas necesarias en el país en cuestión para restablecer su sostenibilidad.

No obstante, su configuración acentúa una serie de problemas: el sistema desigual de ponderación de votos y su efecto negativo sobre la aplicación de las medidas macroeconómicas, la inmunidad del conjunto del MEDE frente a los poderes estatales de los países miembros, la posible falta de viabilidad en el proceso de su auditoría, el agravamiento del déficit democrático y de la legitimidad democrática del conjunto de la Unión Europea, así como el rango reducido de instrumentos y el presupuesto insuficiente del que dispone son solamente algunos de ellos.

Debido a esta situación, el establecimiento de un sistema de gobernanza económica más sólido y persistente con el objetivo de nivelar los desajustes económicos causados por la crisis y con el MEDE exento de los problemas 
marcados, parece ser de máxima actualidad e importancia.

Por último, retomando el estudio de Majone (2003) podemos decir que el problema de déficit democrático de la Unión Europea a futuro se concreta en un aumento de aquel si analizamos la legitimidad procedimental (in) y la legitimidad de resultado (out). En el caso de que la tesis de Scharpf (2000) sea correcta, la brecha entre los ciudadanos y el sistema de gestión política comunitaria se ampliará. Asimismo, si Moravcsik (2002) está en lo cierto, los ciudadanos de los Estados miembros asumirán dicho déficit de legitimidad, siempre y cuando los beneficios de la delegación superen los costes, cosa que por el momento no parece estar ocurriendo.

\section{REFERENCIAS}

Banco Central Europeo (2011). Boletín mensual. Recuperado de http://www.bde.es/f/webbde/SES/ Secciones/Publicaciones/PublicacionesBCE/ BoletinMensualBCE/11/Fich/bm1107.pdf

Barrett, G. (2011). First Amendment? The Treaty Change to Facilitate the European Stability Mechanism. Institute of International and European Affairs.

Beetham, D. (1991). The legitimation of power. Humanities Press International.

Caballero Miguez, G. (2011). Reformas institucionales de la gobernanza económica internacional en tiempos de cambio: debate de ideas, instituciones y política económica. Fundación Alternativas (61).

Castells, M. (1999). La era de la información: economía, sociedady cultura. El poder de la identidad. Madrid: Alianza Editorial.
Dahl, R. A. (1999). Can international organizations be democratic? A sceptic's view. En Shapiro, I. y Hacker-Cordón, C. (eds.). Democracy's edges (pp. 19-36). Cambridge: Cambridge University Press.

Decisión del Consejo Europeo 2011/199/UE de 25 de marzo de 2011, que modifica el artículo 136 del Tratado de Funcionamiento de la Unión Europea en relación con el Mecanismo de Estabilidad para los Estados miembros cuya moneda es el euro.

Dullien, S. y Schwarzer, D. (2011). Dealing with Debt Crisis in the Eurozone. Evaluations and Limits of the European Stability Mechanism. SWP Research Paper (11). Recuperado de: http://www. swp-berlin.org/fileadmin/contents/products/research_papers/2011_RP11_Dullien_swd_ks.pdf

Follesdal, A. y Hix, S. (2006). Why there is a democratic deficit in the EU: A response to Majone and Moravcsik. Journal of Common Market Studies, 44 (3), 533-562.

Habermas, J. (1975). Legitimation crisis. Boston: Beacon Press.

Held, D. (1995). Democracy and the global order: From the modern State to cosmopolitan governance. Cambridge: Polity Press.

Lipset, M. (1963). Political man: The social bases of politics. New York: Doubleday.

Majone, G. (1996). Regulating Europe. New York-London: Routledge.

Majone, G. (2003). The politics of regulation and European regulatory institutions. En Hayward, J. y Menon, A. (eds.). Governing Europe (pp. 297312). Oxford: Oxford University Press.

Martínez, J. A. y Calvo Tirant lo Blach, J. L. (2012). Adelanto del nuevo libro de Banca y Mercados Financieros. Recuperado de: http://portal.uned. es/pls/portal/docs/PAGE/UNED_MAIN/LAUNIVERSIDAD/UBICACIONES/O5/DOCENTE/JOSE_ANTO- 
NIO_MARTINEZ_ALVAREZ/ESTABILIDAD_FINANCIERA_EN_EUROPA_2OI2.PDF

Mecanismo Europeo de Estabilidad (2012). Preguntas frecuentes sobre el MEDE. Recuperado de: http://www.esm.europa.eu/pdf/FAQ\%20 ESM\%2008102012.pdf

Micossi, S., Carmassi, J. y Pierce, F. (2011). On the Tasks of the European Stability Mechanism. CEPS Policy Brief. Thinking ahead for Europe (235). Recuperado de: http://aei.pitt.edu/30039/1/ PB_No_235_Micossi_et_al_on_the_ESM-2.pdf

Moravcsik, A. (2002). In Defence of the Democratic Deficit: Reassessing Legitimacy in the European Union. Journal of Common Market Studies, 40 (4), 603-624.

Moravcsik, A. (2004). Is there a 'Democratic Deficit' in world politics? A framework for analysis. Government and Opposition, 39 (2), 336-363.

O'Kane, R. (1993). Against legitimacy. Political Studies, 41 (3), 471-487.

Pollack, M. (1997). Delegation, agency and agenda-setting in the European Community. International Organization, 51 (1), 99-134.

Pollack, M. (2003). The engines of integration? Delegation, agency and agenda setting in the European Union. Oxford: Oxford University Press.

Rogowski, R. (1974). Rational legitimacy: A theory of political support. Princeton: Princeton University Press.
Scharpf, F. W. (2000). Gobernar en Europa: ¿Eficaz y democráticamente? Madrid: Alianza.

Scharpf, F. W. (2005). Conceptos de legitimación más allá del Estado-nación. Revista Española de Ciencia Politica (13), 13-50.

Tallberg, J. (2000). The anatomy of autonomy: An institutional account of variation in supranational influence. Journal of Common Market Studies, 38 (5), 843-864.

Tallberg, J. (2002). Delegation to supranational institutions: Why, how, and with what consequences? West European Politics, 25 (1), 23-46.

Thatcher, M. y Stone Sweet, A. (2002). Theory and practice of Delegation to non-mayoritarian institutions. West European Politics, 25 (1), 1-22.

Tratado Constitutivo del Mecanismo Europeo de Estabilidad. Recuperado de: http://boe.es/boe/ dias/2012/10/04/pdfs/BOE-A-2012-12378.pdf

Tratado de Lisboa por el que se modifican el Tratado de la Unión Europea y el Tratado constitutivo de la Comunidad Europea, firmado en Lisboa el 13 de diciembre de 2007. Diario Oficial, C-306 de 17 diciembre 2007.

Weber, M. (1964). Economía y sociedad. México: Fondo de Cultura Económica. 